\title{
A rare approach? Microsurgical breast reconstruction after severe burns
}

\author{
Laurenz Weitgasser, Ali Bahsoun, Amro Amr, Michael Brandstetter, Friedrich Knam, \\ Thomas Schoeller
}

Department of Hand, Breast, and Reconstructive Microsurgery, Marienhospital Stuttgart, Stuttgart, Germany

Breast deformity, in post-burn patients, is a common problem leading to lower self-esteem and reclusive behavior that impairs quality of life. The authors present the course of treatment of an 18-year-old immigrant girl who suffered second- to third-degree burns over approximately $20 \%$ of her total body surface area in her early childhood. The second- to third-degree burns were located on her right trunk and abdomen, as well as her right shoulder, neck, and right groin area. Since it was not offered in her home country, reconstructive surgery, including microsurgical breast reconstruction, was sought abroad. Due to the lack of available skin and soft tissue, a bilateral breast reconstruction with free transverse myocutaneous gracilis flaps was offered. This case illustrates one method of using microsurgery to address post-burn breast deformities in order to alleviate psychological suffering and improve quality of life.

Keywords Reconstructive surgery / Burns / Microsurgery / Free-tissue flaps / Gracilis muscle
Correspondence: Laurenz Weitgasser Department of Hand, Breast, and Reconstructive Microsurgery, Marienhospital Stuttgart, Teaching Hospital of the University of Tuebingen, Boeheimstr 37, 70199 Stuttgart, Germany

Tel: +49711-6489-7216

Fax: +49711-6489-2222

E-mail: laurenz.weitgasser@vinzenz.de

This case report was presented at the Mayo Clinic Chang Gung Symposium Reconstructive Surgery on October 19-22, 2016, in Munich, Germany.

Received: 15 Jun 2017 • Revised: 1 Aug 2017 • Accepted: 16 Aug 2017

pISSN: 2234-6163 • elSSN: 2234-6171 • https://doi.org/10.5999/aps.2017.01039• Arch Plast Surg 2018;45:180-184

\section{INTRODUCTION}

Next to burn injuries of the upper extremities, injuries of the trunk are the most common. In young female adolescents, such injuries can impede breast growth and development through excessive scar contracture, leading to hypoplasia as well as displacement of the nipple-areolar complex [1,2]. In addition to asymmetry and impaired aesthetics, post-burn breast deformities cause functional problems in patients' daily life (e.g., trunk movement, breast feeding, and intimacy) [2,3]. Severe burn injuries of the female breasts are challenging to treat, and most surgical approaches offer limited satisfactory aesthetic results.

Various operative techniques have been used to address postburn breast deformities, including tissue expansion and silicone implants, as well as using skin grafts, artificial dermal substitutes, or local muscle flaps in combination with symmetrizing techniques such as mastopexy or reduction mammoplasty [4-8]. Free-tissue reconstruction has been suggested as an alternative technique, but to our knowledge, there are no experiential reports of larger case series where this technique was used $[9,10]$.

Although routinely performed in many plastic surgery units, microsurgical breast reconstruction remains a sophisticated surgical procedure that requires careful planning, subsequent follow-up, as well as possible corrective surgery to achieve a satisfactory functional and aesthetic result.

The case presented here presents the course of treatment of a rarely described bilateral one-stage breast reconstruction with free transverse myocutaneous gracilis (TMG) flaps after a severe burn injury. The authors discuss the advantages and disadvantages of such complex operative approaches and then rec- 
ommend using microsurgical techniques for post-burn breast reconstruction.

\section{CASE}

An 18-year-old girl who suffered second- to third-degree burns over $20 \%$ of her total body surface area after an automobile accident 14 years ago presented at our clinic. She had undergone previous multiple scar revisions and contracture releases in order to correct the burn scar contracture and was seeking further reconstructive treatment.

The girl presented with severe scar contractures that mainly affected her posture, but also affected the movement of her head and neck, resulting in abduction of her right arm (Fig. 1). In addition to functional impairment, she complained of social isolation and avoidance behavior caused by the physical disfigurement of her breasts.

A very rigid scar was present, spanning both breasts and resulting in symmastia and a downward shift of the right inframammary fold by about $3 \mathrm{~cm}$ relative to the left side. There were vestigial remains of the nipple-areolar-complex on the right breast and a complete absence of the nipple-areolar complex on the left breast.

Severely contracted burn scars were found on the right side of the neck and on the right trunk down to the abdomen and right groin. Scar contraction impaired the reclination of the head and abduction of the right arm, as well as resulting in an inclined posture.

Two separate operations were conducted to recover adequate head, neck, and right arm mobility, and a third operation was conducted for breast reconstruction. During the first operation, the scar contractions of the neck and right axillary region were treated with Z-plasties. Split skin grafting was performed to release the contracture of the right flank and to improve the patient's posture, and a tissue expander was implanted in the right parascapular region. After sufficient tissue expansion, we performed the second operation. During this procedure, a free parascapular flap was harvested to improve and reconstruct neck movement and reclination, and plastic surgical reshaping and remodeling of both breasts with local skin flaps was performed to treat the symmastia. In the third operation, one-stage bilateral breast reconstruction with bilateral free TMG flaps, in a modi-

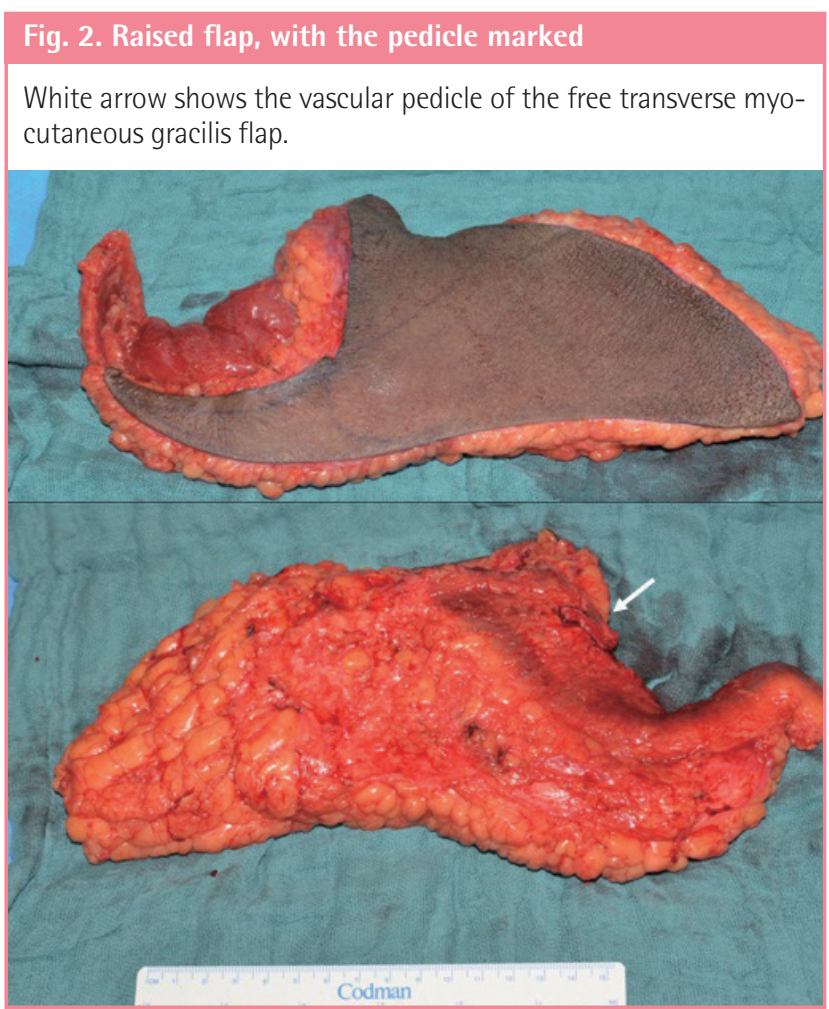

Fig. 1. 18-year-old patient during first consultation

Preoperative appearance, with scarring of both breasts. (A) Front view, (B) lateral view.
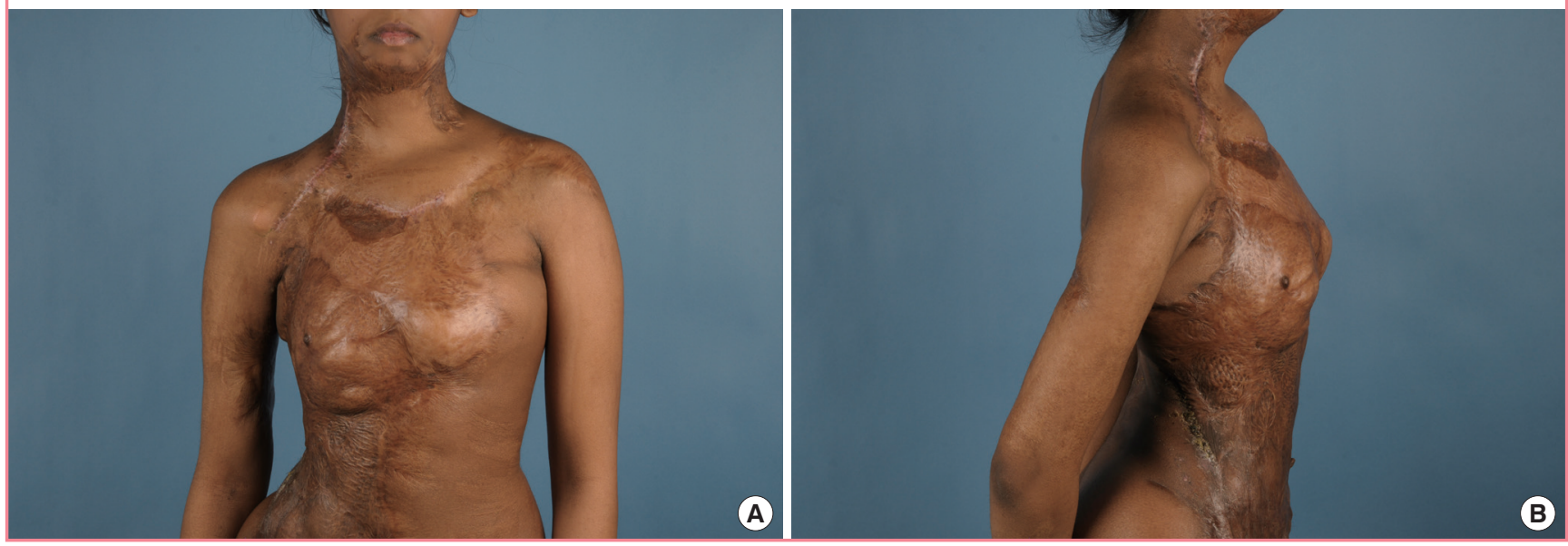
Fig. 3. Bilateral breast reconstruction with free TMG flaps

Intraoperative view of the bilateral transverse myocutaneous gracilis (TMG) flap harvest.

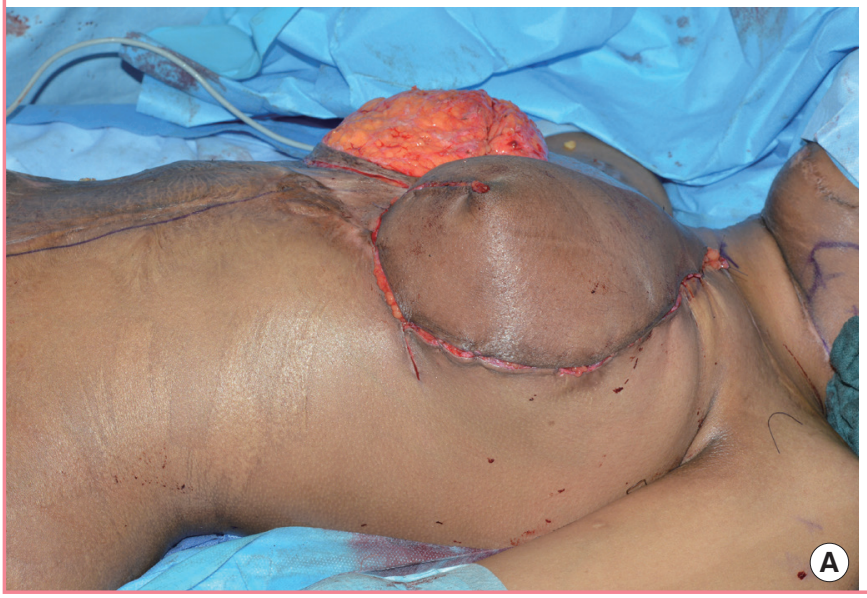

Fig. 4. Final operative result after wound closure

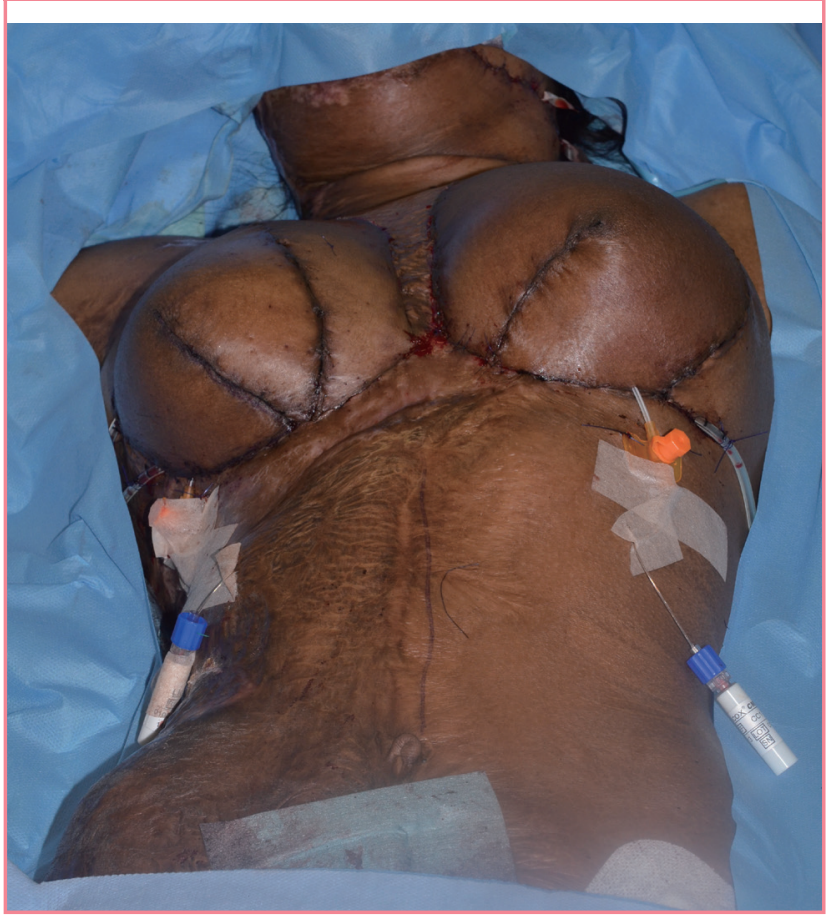

fied fashion, was performed. The operation involved 2 teams of surgeons. One team elevated the flaps (Fig. 2) while the second team prepared the recipient vessels (Fig. 3). The operation lasted approximately 7 hours and 48 minutes. In addition to skinperfusion evaluation and handheld Doppler auscultation, 2 Integra Licox (Integra LifeSciences Co., Saint Priest, France) monitoring devices were used to postoperatively monitor the flaps (Fig. 4).

Unfortunately, the healing course was not totally free from complications. Bilateral partial flap necrosis and wound infec-

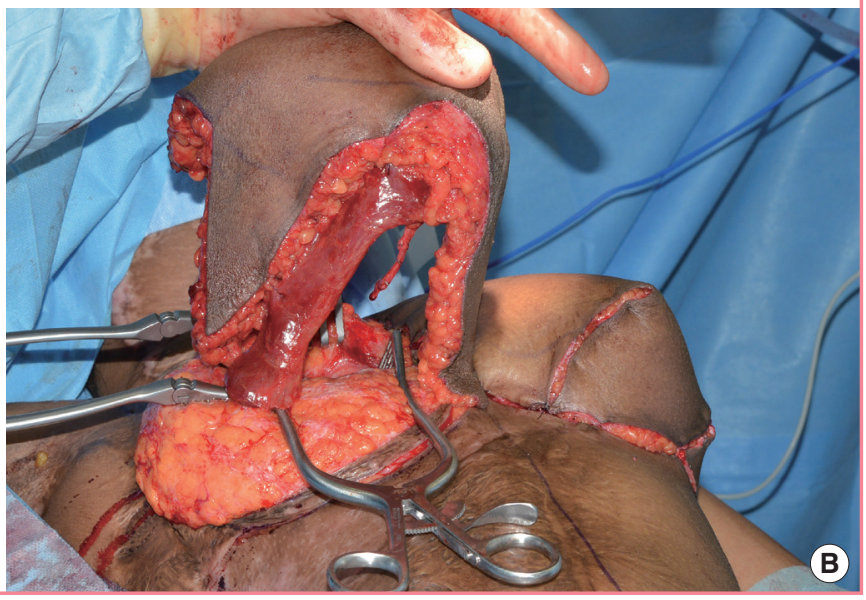

tion had to be addressed with further debridement and negative-pressure wound therapy. Consequently, parts of the left lateral and right medial breast required split skin grafting, which was performed to accelerate wound healing.

Approximately 4 weeks after surgery, an asymmetry of both breasts was still visible, which was easily corrected with autologous fat grafting/lipofilling.

By the last follow-up, 8 weeks after surgery, the patient was satisfied with the results, including symmetrically reconstructed breasts with a newly formed breast mound and a nipple-areolar complex (Fig. 5).

\section{DISCUSSION}

Although autologous breast reconstruction with free flaps, such as deep inferior epigastric perforator (DIEP) or TMG flaps, has become a safe and reliable procedure for breast cancer patients who have undergone a mastectomy or breast-conserving therapy, it generally has not been used to treat post-burn breast deformities. To the authors' knowledge, it has not been used routinely for post-burn corrective surgery. Although microsurgical breast reconstruction is mentioned in the literature $[9,10]$, reconstructive breast surgery most often is limited to techniques such as tissue expansion and silicone implants, as well as using skin grafts, artificial dermal substitutes, or local muscle flaps in combination with symmetrizing techniques of mastopexy or reduction mammoplasty [4-8].

The use of free flaps in early and late burn reconstruction has been described in numerous reports. However, this most often was limited to extremity reconstruction or contracture release of the neck. In the series of Shen et al. [11] series describing 70 free flaps in 65 patients, 43 flaps were in the upper extremity, 16 


\section{Fig. 5. Eight-week postoperative result}

(A) Front view, (B) lateral view.
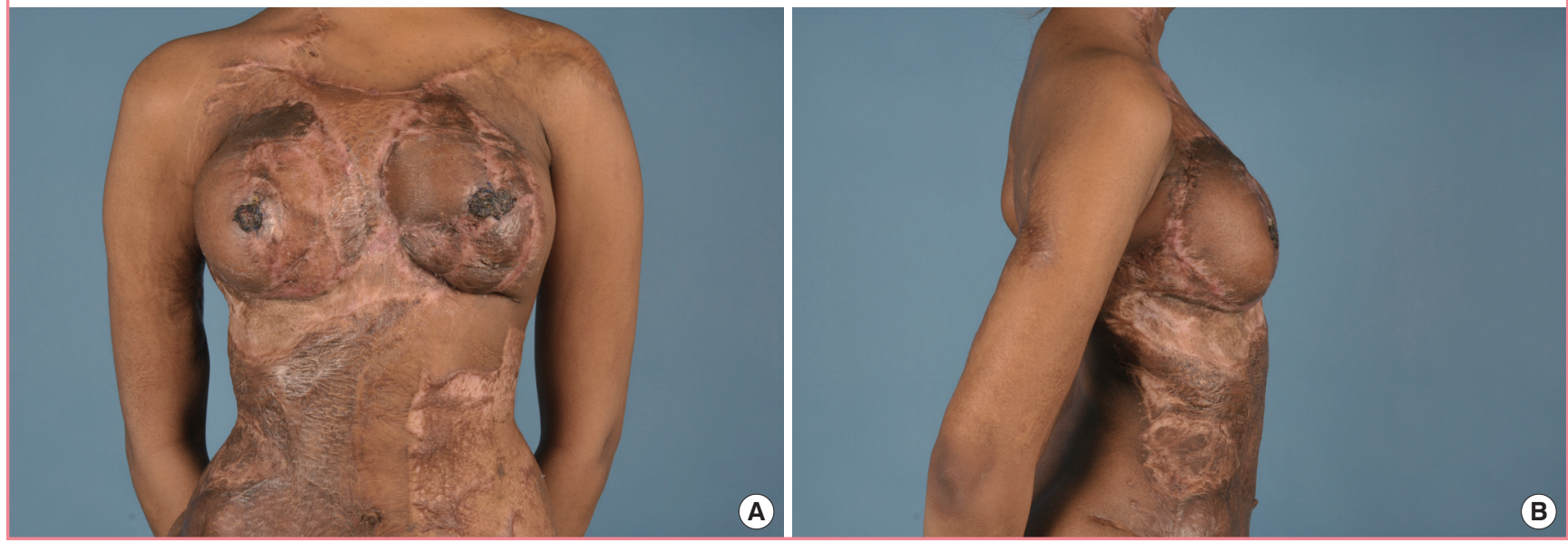

flaps were in a lower limb, and 11 flaps were in the head or neck. De Lorenzi et al. [12] in their series described 53 free flaps in 39 patients, including 27 flaps in an upper limb, 11 flaps in a lower limb, 14 flaps in the head or neck, and only 1 free flap used for thoracic contracture release. Neither series included breast reconstructions. In the large series of Neale et al. [1] of 157 breast reconstructions in burn patients, free flaps were not used at all. This also was the case for Grishkevich's [13] series of 13 breast reconstructions. Overall, it appears that there is limited experience using microsurgical techniques for post-burn breast reconstruction.

As is the case for all plastic surgery, the aim of post-burn reconstructive breast surgery is an aesthetic, often symmetrical, and if possible, also functional result. Depending on the rigidity of the scar contraction, in most cases, the previously mentioned conventional surgical techniques are sufficient to achieve good reconstructive results.

However, we believe that, in certain patients, the potential risk of a free flap transfer can be justified by its unique advantages. Today, microsurgical tissue transfer is a standard procedure, with the risk of free-flap loss and serious complications ranging between $1 \%$ and $5 \%$. The potential benefit of replacing an extensive rigid scar with healthy, unscarred tissue from a free flap outweighs the risk of free-flap compromise or failure. We believe that, in certain cases, free-flap breast reconstruction is a suitable option for post-burn patients with severe breast deformities and sometimes is superior to conventional surgical techniques for post-burn breast reconstruction.

Skin grafting or using artificial dermal substitutes $[5,13]$ are valuable methods to improve scarring and breast shape; however, the received tissue softness and quality are not comparable to unscarred skin and elastic subcutaneous tissue. Local flaps, or advancement flaps after tissue expansion $[8,14]$ of previously non-scarred tissue, are also useful alternatives to free flaps; however, in patients with large surface area burns of the trunk, dorsum, and abdomen, where no local unscarred tissue is present, microsurgery can be a unique alternative approach to improve the shape, softness, and release of scar contractures of the breast. Apart from the observed complications in the present case, onestage bilateral breast reconstruction with TMG flaps offers a good and safe alternative for post-burn breast reconstruction. Other free flaps, such as DIEP flaps, transverse rectus abdominis myocutaneous flaps, or anterolateral thigh flaps, are also useful alternatives, depending on the location of the unscarred donor site and the circumstances of the individual case. Considering the relatively high patient population with post-burn breast deformities, free-tissue transfer for breast reconstruction should be seen as a viable therapeutic option yielding a reconstructive quality hardly achievable by conventional surgical methods. In most cases, this approach is considered a last option, used only when other reconstructive techniques are no longer applicable. For instance, in patients with heavily scarred trunk, back, and abdominal areas, unscarred tissue sources can only be accessed via free-tissue transfers. However, these observations are not reflected in the literature.

In summary, analogous to post-cancer patients, post-burn patients should be made aware of all possible methods of breast reconstruction that are available to them. More cases of open microsurgical breast reconstruction need to be reported and evaluated to confirm its benefits and eventually lead to it becoming a standard therapeutic option for treating post-burn breast deformities. 


\section{NOTES}

\section{Conflict of interest}

No potential conflict of interest relevant to this article was reported.

\section{Ethical approval}

The study was performed in accordance with the principles of the Declaration of Helsinki. Written informed consents were obtained.

\section{Patient consent}

The patients provided written informed consent for the publication and the use of their images.

\section{ORCID}

Laurenz Weitgasser https://orcid.org/0000-0001-9629-2228

\section{REFERENCES}

1. Neale HW, Smith GL, Gregory RO, et al. Breast reconstruction in the burned adolescent female (an 11-year, 157 patient experience). Plast Reconstr Surg 1982;70:718-24.

2. Robert RS, Blakeney PE, Meyer WJ 3rd. Impact of disfiguring burn scars on adolescent sexual development. J Burn Care Rehabil 1998;19:430-5.

3. Ogilvie MP, Panthaki ZJ. Burns of the developing breast. J Craniofac Surg 2008; 19:1030-3.

4. MacLennan SE, Wells MD, Neale HW. Reconstruction of the burned breast. Clin Plast Surg 2000;27:113-9.

5. Tsoutsos D, Stratigos A, Gravvanis A, et al. Burned breast reconstruction by expanded artificial dermal substitute. J Burn Care Res 2007;28:530-2.

6. Guan WX, Jin YT, Cao HP. Reconstruction of postburn female breast deformity. Ann Plast Surg 1988;21:65-9.

7. Ozgur F, Gokalan I, Mavili E, et al. Reconstruction of postburn breast deformities. Burns 1992;18:504-9.

8. Slator RC, Wilson GR, Sharpe DT. Postburn breast reconstruction: tissue expansion prior to contracture release. Plast Reconstr Surg 1992;90:668-71.

9. Bayram Y, Sahin C, Sever C, et al. Custom-made approach to a patient with post-burn breast deformity. Indian J Plast Surg 2014;47:127-31.

10. Iwuagwu FC, Wilson D, Bailie F. The use of skin grafts in postburn contracture release: a 10-year review. Plast Reconstr Surg 1999;103:1198-204.

11. Shen TY, Sun YH, Cao DX, et al. The use of free flaps in burn patients: experiences with 70 flaps in 65 patients. Plast Reconstr Surg 1988;81:352-7.

12. De Lorenzi F, van der Hulst R, Boeckx W. Free flaps in burn reconstruction. Burns 2001;27:603-12.

13. Grishkevich VM. Restoration of the shape, location and skin of the severe burn-damaged breast. Burns 2009;35: 1026-35.

14. Haik J, Grabov-Nardini G, Goldan O, et al. Expanded reverse abdominoplasty for reconstruction of burns in the epigastric region and the inframammary fold in female patients. J Burn Care Res 2007;28:849-53. 